\title{
Análisis de sentimiento en Instagram: polaridad y subjetividad de cuentas infantiles
}

\author{
Sentimenduen analisia Instagramen: \\ haurren kontuen polaritatea eta subjektibotasuna \\ Sentimental analysis in Instagram: \\ polarity and subjectivity of children's accounts
}

\author{
Arantxa Vizcaino-Verdu, Ignacio Aguaded $\star$ \\ Universidad de Huelva
}

\begin{abstract}
RESUMEN: Instagram se ha convertido en parte integral de la vida cotidiana de niños y jóvenes, gestando una suerte de álbum familiar. En este escenario, analizamos la polaridad y subjetividad de 772 entradas de texto de cuentas infantiles gestionadas por padres en la plataforma mediante procesamiento del lenguaje natural con machine learning y análisis de contenido. Los resultados revelaron una prominente positividad y subjetividad en el campo léxico de cuatro cuentas en español y cuatro en inglés, con el reiterado empleo de los adjetivos feliz, nuevo, súper, etc. En suma, las cuentas infantiles expresan una tendencia de bucólica y festiva crianza.
\end{abstract}

PALABRAS CLAVE: Instagram; infancia; análisis de sentimientos; polaridad; subjetividad; felicidad.

ABSTRACT: Instagram has become an integral part of the daily lives of children and young people, creating a kind of family album. In this context, we analyzed the polarity and subjectivity of 772 text posts in children's accounts managed by parents on the platform through natural language processing with machine learning and content analysis. The results revealed a prominent positivity and subjectivity in the lexical field of four accounts in Spanish and four in English, with the repeated use of the adjectives happy, new, super, etc. In short, the children's accounts express a tendency towards bucolic and festive upbringing.

KEYWORDS: Instagram; childhood; analysis of sentiments; polarity; subjectivity; happiness.

\footnotetext{
* Correspondencia a / Corresponding author: Ignacio Aguaded. Universidad de Huelva. Facultad de Ciencias de la Educación. Campus El Carmen. Avda Tres de Marzo, s/n (21071 Huelva). Spain - aguaded@uhu.es - https://orcid.org/0000-0002-0229-1118

Cómo citar / How to cite: Vizcaino-Verdu, Arantxa; Aguaded, Ignacio (2020). «Análisis de sentimiento en Instagram: polaridad y subjetividad de cuentas infantiles", Zer, 25(48), 213-229. (https://doi.org/10.1387/zer.21454).

Recibido: 01 junio, 2019; aceptado: 29 marzo, 2020.

ISSN 1137-1102 - eISSN 1989-631X / (c) 2020 UPV/EHU

(c) (i) Esta obra está bajo una licencia

Creative Commons Atribución 4.0 Internacional
} 


\section{Introducción}

Las redes sociales se han convertido en un espacio de transformación, divulgación y expresión social, donde se refleja consciente e inconscientemente la visión del mundo individual por medio de personalidades, ideologías y experiencias (CalvoGonzález \& San-Fabián-Maroto, 2018). Un medio y forma de vida ubicua propia del siglo XXI, donde los smartphones y multiplicidad de escenarios en línea facilitan una constante conexión (Marinas, 2019). En este entorno multimodal nacía en 2010 Instagram, una plataforma esencialmente visual ideada por Kevin Systrom y Mike Krieger, cuyo fin último es compartir fotografias y vídeos editados desde cualquier lugar, en cualquier momento (Instagram, 2020).

Tal y como afirman Casado-Riera y Carbonell (2018) y Lee, Lee, Moon y Sung (2015) en referencia a las relaciones sociales en línea, una de las gratificaciones sustanciales de Instagram es socializar con otros usuarios (amigos y comunidades de seguidores) que comparten intereses comunes. El número de cuentas de extraños de las que se es seguidor puede generar depresión, en opinión de Lup, Trub y Rosenthal (2015), quienes consideran negativa estas interacciones. De hecho, DeCasas-Moreno, Tejedor-Calvo y Romero-Rodríguez (2018) afirman que una de las temáticas preferentes en este medio se vincula a los asuntos cotidianos y a la vida personal. De modo que la documentación visual en la red contribuye a la presentación y preservación de la identidad de una persona (Hummel-O’Donnell, 2018). Asimismo, estos entornos son capaces de reforzar las experiencias vitales, influyendo en el auto-concepto y proveyendo, en algunos casos, de felicidad (Berezan, Krishen, Agarwal \& Kachroo, 2017).

Como resultado de este contexto y, partiendo de la concepción multimodal y positivista de Instagram, donde la escritura forma parte intrínseca del elemento visual (Domingo, Jewitt \& Kress, 2015), se presenta un estudio exploratorio que profundiza en la expresión del yo infantil a través de la escritura vernácula. Se trata de iniciar una aproximación a la reafirmación de la identidad infantil, con el objetivo de identificar el grado de polaridad y subjetividad expresado a nivel léxico, basándose en la hipótesis de que el cuadro textual de las cuentas infantiles gestionadas por los padres en Instagram proyecta un halo de positividad y felicidad idílica.

\section{Idealización de la infancia en Instagram}

Uno de los cambios exponenciales en el crecimiento de las redes sociales, y en concreto de Instagram, es el espacio en el que se han visto envueltos niños y jóvenes, y en cómo estas plataformas se han convertido en parte integral de sus vidas (Livingstone, Mascheroni, Ólafsson \& Haddon, 2014; Hadjipanayis et al., 2019; 
Willoughby, 2018). En la era de la digitalización contemporánea, los niños interactúan con tablets, dispositivos móviles y redes sociales como medio de consumo y producción multimodal, de modo que, en 2017, el 23\% de entre 8-11 años y el $74 \%$ de entre 12-15 años tenían acceso por lo menos a una red social; y a partir de los 12 años, la mitad contaba con perfiles propios (Wenholm \& Reneland-Forsman, 2019). En estos casos, como indican Díaz-Bustamante y Llovet-Rodríguez (2017), se genera una transición vertiginosa de la infancia a la adolescencia, auspiciada por alteraciones fisicas y desórdenes psicológicos.

En efecto, Hadjipanayis et al. (2019), declaran que el impacto de las redes sociales sobre los niños presenta un cuadro tanto positivo como negativo. El primero asociado a la mejora de socialización y comunicación, desarrollo de competencias y aprendizaje informal (Vizcaíno-Verdú, Contreras-Pulido \& Guzmán-Franco, 2019; Izquierdo-Iranzo \& Gallardo-Echenique, 2020). El segundo, adscrito a riesgos potenciales como la falsificación de la edad e identidad, el ciberacoso (Larrañaga, Navarro \& Yubero, 2018), el sexting (Chacón-López, Romero-Barriga, Aragón-Carretero \& Caurcel-Cara, 2016) la depresión, glamourización, ciberostracismo (Peña-Cárdenas, Rojas-Solís \& García-Sánchez, 2018), la privacidad y los trastornos del sueño. Esta influencia viene dada, en la mayoría de los casos y, tal y como apuntan Ho, Shin y Lwin (2017), a la autoridad de los padres. Y es que, según la American Psychological Association (2007), una de las razones que motiva la introducción de las redes sociales en la vida diaria infantil son los padres.

La mediación parental en este entorno ha demostrado que la familia puede mitigar o potenciar los efectos mediáticos sobre los niños, tales como la agresión inducida por medios de comunicación, el materialismo inducido por la publicidad (Gómez-Espino \& Blanco-López, 2005) y el consumo de alimentos no saludables (Nathanson, 2004; Buijzen, Schuurman \& Bomhof, 2008). Más aun, esta influencia en escenarios como el de Instagram ha sido ampliamente estudiada desde el concepto visual, acuñándose como sharenting u oversharenting: véase compartir información (o demasiada información) sobre hijos en entornos virtuales. Fenómeno a menudo criticado por el latente motivo oculto de ganar dinero a costa a los infantes (Choi \& Lewallen, 2017; Matijaščić, 2018).

Le-Moignan, Lawson, Rowland, Mahoney y Briggs (2017) atienden a este respecto a las instantáneas familiares como un remanente histórico de la fotografia Polaroid, a partir de la cual se reproducía públicamente la cotidianeidad familiar, y donde los niños eran modelo de popularidad y atracción, tal y como sucede en Instagram (Choi \& Lewallen, 2017). Estos álbumes cumplimentados de instantáneas, señala Rose (2010), han sido estructurados y generados conscientemente como un tipo de memoria distorsionada. $\mathrm{O}$ expresado de otro modo, por omisión y elección selectiva de lo registrable en cuanto a composición, personas, etc., estas fotografias son ex- 
puestas en una aparente aura de felicidad, fotogenia y romántica crianza, implacablemente optimista de la vida familiar (Bourdieu, 1990).

\section{Positividad y felicidad en redes sociales}

Diener (2009) y Veenhoven (1991) describen la felicidad como la auto-evaluación de la calidad de vida. El primero profundiza y acentúa en esta como una suerte de bienestar subjetivo, donde intervienen una parte afectiva (agrado o desagrado) y una cognoscitiva (satisfacción general o específica). Si bien, la felicidad puede convertirse en una meta inabarcable para los individuos de una sociedad digitalizada y capitalista, donde esta queda supeditada a la adquisición de bienes y servicios (Berezan et al., 2017).

En efecto, las redes sociales en general, e Instagram en particular, son productos experienciales que refuerzan continuamente hábitos positivos y/o negativos (Scheinbaum, 2017). Bosangit y Demangeout (2016) indican que muchas de estas plataformas facilitan a sus usuarios el consumo de experiencias, generando auto-reflexión, crecimiento y aprendizaje y, en muchos casos, potenciando el grado de felicidad. Por lo que no es de extrañar que autores como Gorton y Garde-Hansen (2013) subrayen la noción de Internet y sus correspondientes escenarios en línea como un medio de uso terapéutico. Chae (2018) introduce, además, que el uso activo de estos espacios suscita una especie de felicidad en la construcción de capital y conexión social.

Varios estudios apuntan hacia el sesgo de esta positividad en línea, en tanto que la mayor parte de contenidos distribuidos en redes sociales obtienen una valoración más positiva que negativa (Reinecke \& Trepte, 2014; Waterloo, Baumgartner, Peter \& Valkenburg, 2018). Se convierten, en este orden de cosas, en contenidos agradables generados por el usuario, donde este adopta el rol de experto en felicidad, gestando una comuna de oportunidades y proyectos felices (Dale et al., 2019; Gorton \& Garde-Hansen, 2013). Una idea que delimita cadenas de experiencias y emociones guiadas por la interacción con la familia, los amigos y otros usuarios en pos de la auto-concepción (Freitas, 2017).

En el contexto de las emociones y el positivismo en Internet, se encuentran investigaciones que abogan por la comunicación computacional y la expresión de emociones a través de redes sociales, de las que se desprenden una vinculación directa entre lenguaje emocional y comportamiento online (Dresner \& Herring, 2010; Huffaker, 2010). Destacan, por ejemplo, exploraciones sobre las técnicas de visión computacional en Instagram para definir asociaciones entre personalidad y género por medio de la fotografia (Kim \& Kim, 2018); análisis de emociones de los usuarios frente a las marcas en redes sociales (Diayanah-Abdullah \& Asnira-Zolkepi, 2017); y análisis de sentimiento en Instagram de lectores de biblioteca (Zhan, Tu \& Yu, 
2018). En virtud de este marco, se introduce el presente análisis, que trata de amplificar los estudios relativos al análisis de sentimiento semántico en Instagram y, en concreto, al grado de positividad originado en cuentas de infantes.

\section{Método y materiales}

Para el desempeño de la presente investigación se aplicó una metodología cuanti-cualitativa por medio de la minería de datos y el análisis de contenido semántico. La primera, según Ao (2018), da paso a un gran número de algoritmos ocultos empleados hasta la fecha por diferentes áreas (marketing, publicidad, salud, ingeniería e informática) sustentadas en la estadística, el procesamiento de la información, el aprendizaje automático y el reconocimiento de patrones.

Desde esta área, el análisis de sentimiento pretende estudiar desde el ámbito informático-informacional expresiones preferentes y personales, también conocidas como minería de emociones y extracción de opiniones (Araque, Corcuera-Plastas, Sánchez-Rada \& Iglesias, 2017). El fin último de esta técnica es identificar factores sentimentales en tendencia a través de la expresión semántica y la representación sintáctica, combinando polaridad y subjetividad. Una herramienta apta para la exploración de redes sociales, así como para la detección de nuevos patrones comportamentales (Micu, Micu, Geru \& Lixandroiu, 2017). Según Ashok, Rajanna, Joshi y Kamath (2016), este instrumento aplica un sistema de procesamiento del lenguaje natural o $\mathrm{Na}$ tural Language Processing (NLP), que identifica y extrae información lingüístico-computacional de fuentes preexistentes. Este medio se emplea para el análisis de ingentes cantidades de información, considerándose un sistema de clasificación de opiniones y sentimientos en texto cada vez más cotizado (Liu, 2015). Las aplicaciones dominantes sobre análisis de sentimiento se basan, fundamentalmente, en machine learning: máquina o software capaz de aprender automáticamente por medio de algoritmos e informaciones previamente introducidas en su sistema (Pang, Lee \& Vaithyanathan, 2002).

El análisis de contenido (Krippendorff, 1980), simultáneamente, facilitó la extracción de adjetivos recurrentes en las entradas de texto observadas, para la comparación mediante análisis computacional y léxico del carácter positivo de las cuentas infantiles. La justificación de la selección de adjetivos frente a sustantivos y otras construcciones radica de la propuesta de Asch (1946), por la que a partir de estos se describe necesariamente la personalidad de un individuo.

\subsection{INSTRUMENTOS DE INVESTIGACIÓN}

El software empleado para el análisis de sentimientos es TextBlob. Una librería modular de acceso abierto y procesamiento de texto diseñada por Python, que pro- 
porciona una API de observación NLP, y que posibilita el etiquetado de partes de la voz, extracción de sustantivos, análisis de sentimientos, etc. (Ao, 2018; Hasan, Moin, Karim \& Shamshirband, 2018). Esta herramienta, dispuesta como instrumento de la investigación principal, provee de las características básicas de los fundamentos NLP mediante código Python, que permiten analizar y calcular la polaridad y subjetividad de un texto en español e inglés.

El modo de aplicación se centra en el factor de polaridad en una escala de -1 (muy negativo) a 1 (muy positivo), donde 0 se delimita como texto neutro. Asimismo, se aplica al factor subjetividad una escala de 0 a 1 , donde 0 es muy objetivo y 1 muy subjetivo. Para el análisis de contenido se han aplicado el lector inmersivo de Outlook en la identificación de adjetivos en español/inglés, y el software gratuito en línea WordCounter para la localización de los ítems más frecuentes.

\subsection{Muestra}

Dadas las posibilidades de análisis del cuerpo de texto en español e inglés en TextBlob, el lector inmersivo de Outlook y WordCounter, se seleccionó una muestra de ocho cuentas infantiles (772 entradas de texto) de Instagram (cuatro en español y cuatro en inglés). Estas se delimitaron a partir de la plataforma analítica HypeAuditor, por la que se extraen influencers tanto de YouTube como Instagram. La plataforma recomendó ocho cuentas de instagramers infantiles en español e inglés que, independientemente de su localización, presentaran una media de engagement del 8,29\%, con un rango de edad de entre 1 a 7 años, ajustado a la etapa etaria de la primera infancia (de 0 a 8 años) según la UNESCO (2020).

Las características de la muestra se acogen a los siguientes aspectos: 1) Texto de entrada en español o inglés; 2) Niños y/o niñas como fuente visual principal; 3) Niños y/o niñas de la etapa etaria correspondiente a la primera infancia; 4) Cuenta gestionada por uno/ambos padres/madres explícitamente destacado en el perfil de la cuenta (gestionado por, IG familiar, presentación de la cuenta en plural con implicación familiar). De este criterio, adicionalmente, se añade que las entradas de estas cuentas infantiles se refieren a las actividades de sus protagonistas en tercera persona (por ejemplo: Jamás me imaginé que serías así... eres tan especial!! Te queremos!).

Las unidades de análisis se presentan en la tabla 1, donde se anonimizan los datos personales e identificativos de las cuentas infantiles, en tanto que se proporciona información de menores. El estudio se acoge a los principios éticos del Código de Conducta APA en materia de uso de datos personales infantiles (American Psychological Association, 2017). 
TABLA 1

Muestra de observación

\begin{tabular}{cllccr}
\hline Canal & Idioma & País & $\begin{array}{c}\text { Edad } \\
\text { (años) }\end{array}$ & $\begin{array}{c}\text { Número } \\
\text { de seguidores }\end{array}$ & $\begin{array}{c}\text { Número } \\
\text { de imágenes observadas }\end{array}$ \\
\hline 1 & Español & España & 4 & 242.000 & 100 imágenes \\
2 & Español & España & 3 y 1 & 165.000 & 72 imágenes \\
3 & Español & España & 2 & 59.000 & 100 imágenes \\
4 & Español & España & 5 & 4.471 & 100 imágenes \\
5 & Inglés & EEUU & 7 & 1.200 .000 & 100 imágenes \\
6 & Inglés & Australia & 5 & 824.000 & 100 imágenes \\
7 & Inglés & EEUU & 8 & 589.000 & 100 imágenes \\
8 & Inglés & EEUU & 4 & 32.000 & 100 imágenes \\
\hline
\end{tabular}

\subsection{Procedimiento}

\subsubsection{Fase de recopilación de datos}

El primer paso en la investigación fue recopilar una totalidad de 100 imágenes por cuenta infantil, de modo que se introdujeron a la muestra hasta 772 entradas de texto aleatorias. La razón por la que una cuenta presenta 72 entradas se justifica en la limitación cuantitativa de esta. Esto es, se extrajo el número máximo de entradas disponibles en el momento en que se desarrolló el estudio (de septiembre a noviembre de 2019). De cada entrada, se introdujeron los cuerpos de texto en hoja de cálculo, incluyendo signos de interrogación, puntuación, emoticonos, etc.

\subsubsection{Fase de análisis de sentimiento}

A continuación, y, mediante librería TextBlob, se registraron cada una de las entradas por cuenta infantil en el sistema con una línea de código ajustada al texto en español o inglés, tal y como se presenta en la tabla 2 .

TABLA 2

\section{Línea de código TextBlob}

\begin{tabular}{ll}
\hline \multicolumn{1}{c}{ Español } & \multicolumn{1}{c}{ Inglés } \\
\hline from textblob import TextBlob & from textblob import TextBlob \\
$\mathrm{t}=\mathrm{TextBlob}(\langle »)$ & $\mathrm{t}=\mathrm{TextBlob}(\langle »)$ \\
ten=t.translate $(\mathrm{to}=» e n »)$ & ten=t \\
print $($ ten,» $\backslash \mathrm{n} »)$ & $\operatorname{print}(\mathrm{ten}, » \backslash \mathrm{n} »)$ \\
print $($ ten.sentiment $)$ & print $($ ten.sentiment $)$ \\
\hline
\end{tabular}

Fuente: elaboración propia. 
Cada entrada produjo un resultado de polaridad (muy negativo, neutro o muy positivo) y de subjetividad (muy objetivo o muy subjetivo), recopilado en hoja de cálculo para la extracción del cómputo total.

\subsubsection{Fase de identificación de adjetivos frecuentes}

En última instancia se registraron las entradas de texto en el lector inmersivo de Outlook, identificando y recopilando adjetivos. Seguidamente, se introdujo la lista de adjetivos (primero por cuenta, después por idioma, y finalmente de la muestra total) en WordCounter, a fin de determinar aquellos adjetivos frecuentes en las cuentas infantiles de Instagram.

\section{Resultados}

\subsection{AnÁlisis de SEntimiento De Cuentas infantiles}

Los resultados obtenidos a raíz del registro y procesamiento de las 772 entradas de texto en TextBlob, se analizaron individualmente y en correlación a sus coetáneas en materia de idioma y polaridad, como se contempla en la tabla 3.

TABLA 3

Polaridad en cuentas infantiles en inglés

\begin{tabular}{ccccccc}
\hline Cuenta & $\begin{array}{c}\text { Número } \\
\text { de entradas } \\
\text { positivas }\end{array}$ & $\begin{array}{c}\text { Número } \\
\text { de entradas } \\
\text { negativas }\end{array}$ & $\begin{array}{c}\text { Número } \\
\text { de entradas } \\
\text { neutras }\end{array}$ & $\begin{array}{c}\text { Media de } \\
\text { polaridad } \\
\text { positiva }\end{array}$ & $\begin{array}{c}\text { Media de } \\
\text { polaridad } \\
\text { negativa }\end{array}$ & $\begin{array}{c}\text { Valoración } \\
\text { media de } \\
\text { polaridad } \\
\text { de la cuenta }\end{array}$ \\
\hline 1 & 56 & 8 & 36 & 0,4253727004 & $-0,2203125$ & 0,220583722 \\
2 & 84 & 6 & 10 & 0,3815651739 & $-0,215133759$ & 0,3076067205 \\
3 & 56 & 13 & 31 & 0,279027853 & $-0,194726176$ & 0,1309411947 \\
4 & 83 & 6 & 11 & 0,5002992081 & $-0,142706886$ & 0,4066859295 \\
\hline Total & 279 & 33 & 88 & 0,3965662339 & $-0,19321983$ & 0,2664543917 \\
\hline
\end{tabular}

Fuente: elaboración propia.

Del cómputo de 400 entradas totales referentes a la muestra de habla inglesa, se descubre una media de $69,75 \%$ de entradas positivas, $22 \%$ neutras y $8,25 \%$ negativas. La polaridad derivada de las entradas positivas presenta un valor de $39,65 \%$ y las negativas de $-19,32 \%$. En suma, en esta primera escala, se descubre una polaridad media del $26,64 \%$. 
TABLA 4

Polaridad en cuentas infantiles en español

\begin{tabular}{ccccccc}
\hline Cuenta & $\begin{array}{c}\text { Número } \\
\text { de entradas } \\
\text { positivas }\end{array}$ & $\begin{array}{c}\text { Número } \\
\text { de entradas } \\
\text { negativas }\end{array}$ & $\begin{array}{c}\text { Número } \\
\text { de entradas } \\
\text { neutras }\end{array}$ & $\begin{array}{c}\text { Media de } \\
\text { polaridad } \\
\text { positiva }\end{array}$ & $\begin{array}{c}\text { Media de } \\
\text { polaridad } \\
\text { negativa }\end{array}$ & $\begin{array}{c}\text { Valoración } \\
\text { media de } \\
\text { polaridad de la } \\
\text { cuenta }\end{array}$ \\
\hline 1 & 46 & 4 & 22 & 0,4301262341 & $-0,2640625$ & 0,2601327324 \\
2 & 94 & 6 & 0 & 0,3970490684 & $-0,1748824$ & 0,3627331792 \\
3 & 80 & 7 & 13 & 0,3791975789 & $-0,1728445$ & 0,2912589469 \\
4 & 62 & 18 & 20 & 0,3614976756 & $-0,1805505$ & 0,1916294657 \\
\hline Total & 282 & $\mathbf{3 5}$ & $\mathbf{5 5}$ & $\mathbf{0 , 3 9 1 9 6 7 6 3 9 3}$ & $-\mathbf{- 0 , 1 9 8 0 8 4 9}$ & $\mathbf{0 , 2 7 6 4 3 8 5 8 1 1}$ \\
\hline
\end{tabular}

Fuente: elaboración propia.

Continuando con la muestra española (372 entradas), se traza una media de $75,81 \%$ entradas positivas, $14,66 \%$ negativas y $9,4 \%$ neutras. La polaridad positiva presenta valor medio de 39,19\% y la negativa de 19,8\%. En total, la polaridad de esta segunda muestra es de $27,64 \%$. Por lo que, para ambos casos, y del universo total observado, se contempla una polaridad media del 30,1\%: las cuentas infantiles presentan mayor número de entradas positivas que negativas.

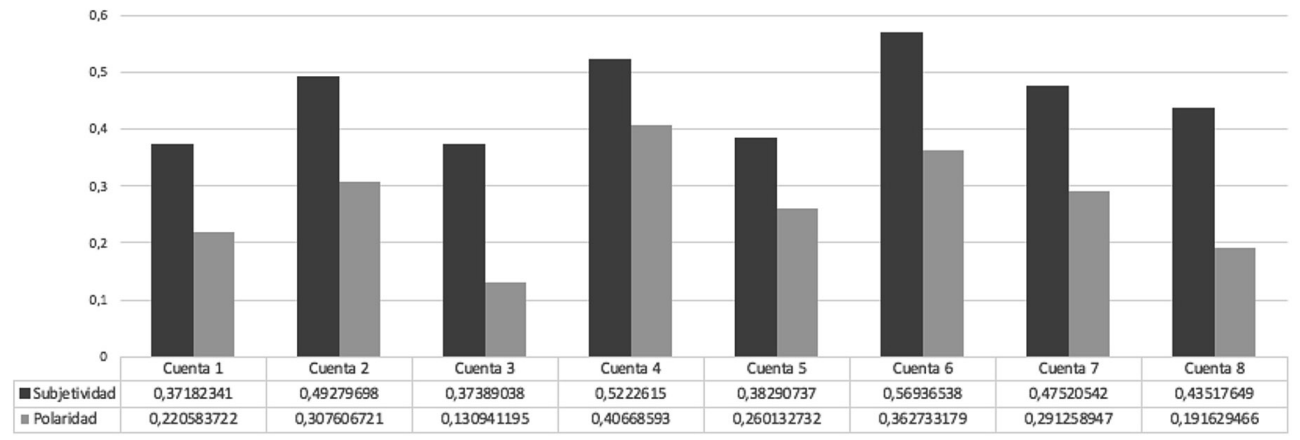

Fuente: elaboración propia.

FIGURA 1

Subjetividad y polaridad en cuentas infantiles de Instagram

En cuanto a la subjetividad, se percibe una afinidad entre las cuentas infantiles inglesas y españolas al resultar un 0,44 y un 0,46 respectivamente en la escala de análisis $(0=$ muy objetivo; $1=$ muy subjetivo). En este orden y, considerando la media de ambas $(0,5)$, se presenta una suerte de neutralidad o, en este caso, de subjetividad media en las cuentas infantiles de Instagram. 
Adicionalmente y, tras comparar en la Figura 1 los resultados relativos a la subjetividad y polaridad, se observa una discordancia entre ambos ejes. Esto es, las entradas subjetivas no son proporcionales al grado de positividad del texto.

\subsection{AnÁlisis De CONTENIDO SEMÁNTICO: ADJETIVOS FRECUENTES}

De la muestra total, se recopilan 1.480 adjetivos (700 en inglés y 780 en español), donde se muestra una densidad correlativa, tal y como se muestra en la Figura 2 .
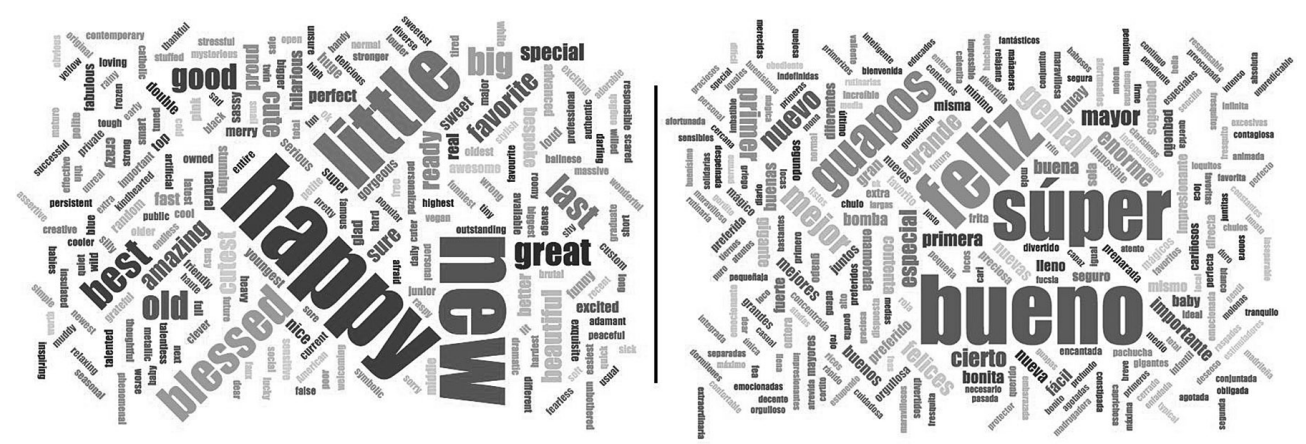

Fuente: elaboración propia.

FIGURA 2

Nube de adjetivos frecuentes en cuentas infantiles de Instagram en español e inglés

En inglés, se localizaron en orden de densidad: 58 palabras del término happy (8\%), 58 de new (8\%), 46 de little (7\%), 26 de blessed (4\%), 21 de best (3\%), y así sucesivamente, hasta reflejar adjetivos únicos (1\%). En español, se compendiaron $47 \mathrm{pa}-$ labras con feliz (8\%), 46 con súper (7\%), 29 con guapos (5\%), 17 con genial (3\%), 15 con nuevo (2\%), entre otras. En ambos casos, se refleja la reiteración de la concepción «feliz» como adjetivo recurrente y frecuente en el léxico de los instagramers infantiles.

\section{Discusión y conclusiones}

Los resultados arrojados tanto por el análisis de sentimiento como por la exploración de adjetivos en las entradas textuales de las cuentas infantiles en Instagram, presentan un grado prominente de positividad (polaridad) en un entorno medianamente subjetivo. En la correlación entre polaridad y subjetividad, no hemos observado niveles pares que indiquen que una respuesta positiva y/o negativa vaya proporcionalmente vinculada al modo en que se expresan los progenitores de los niños. 
Por lo que nos hallamos ante una muestra del género histórico-fotográfico e idílico introducido desde antaño por la instantánea familiar de Polaroid, extrapolada al entorno digital (Rose, 2010; Bourdieu, 1990).

La hipótesis planteada, por la que se describiría un contexto de emociones felices expresadas a través de construcciones semántico-sintácticas, queda ratificada por un $69,75 \%$ y un $75,81 \%$ de entradas de carácter positivo. De hecho, tanto en las cuentas inglesas como en las españolas, el número de comentarios con construcciones semánticas negativas es similar, con una media del $8,25 \%$ y $8,75 \%$ respectivamente. Un fenómeno que se reproduce en la escala de la subjetividad, donde se observa una media de 0,44 y 0,46 puntos. Resulta esencial atender, a raíz de estos datos, cómo el perfil de cuentas que proyectan contenidos e historias de vida desiguales y «exclusivas», así como escenarios geográficos y lenguajes dispares, reflejan un contexto semántico similar. En este punto, se podría apuntar hacia la estandarización o la jerga del instagramer (Berlanga-Fernández \& Martínez-Rodrigo, 2010).

En relación al reiterado uso del adjetivo feliz en ambos segmentos, así como otras construcciones relativas al bienestar y a la satisfacción de los infantes y la familia (nuevo, mejor, súper, genial, especial, increíble, bueno, etc.), observamos un conjunto de términos abocados a un universo en línea onírico. En este sentido, los datos reflejan una concomitancia destacable entre el influencer anglosajón y español, donde se engrandece la figura de niños que no superan los 10 años de edad (mayor, bueno/a, guapo/a, especial, bonito/a, mono/a, etc.), y en el que la persona gramatical predominante es la tercera. Esto es, los padres describen no solo las emociones o sentimientos de sus hijos, sino que detallan su aspecto fisico. Un factor remarcable, en tanto que, en las descripciones de los perfiles, los padres explicitan la gestión de estos, ajenos a las preferencias o al consecuente auto-concepto que niño o niña pueda desarrollar con el tiempo (Berezan et al., 2017; Wernholm \& RenelandForsman, 2019). La cuestión que planteamos a partir de esta situación de trascendencia internacional es, ¿cómo se correlaciona la felicidad con la realidad de las familias en los perfiles? ¿Se está generando una suerte de auto-concepción desigual a la de la realidad, por la que Thurlow, Lengel y Tomic (2004) delimitarían a estos niños con la terminología de identidad digital y no de identidad en línea? Es decir, los infantes parecen presentar una identidad exclusiva (identidad digital) y construida especialmente para la Red.

Entendemos que las circunstancias que favorecen espacios en línea y sociales bucólicos pueden acogerse a la noción de emociones auto-trascendentes expuesta por Diessner, Solom, Frost, Parsons y Davidson (2008), por la que advierten que los contenidos más traslúcidos y cotidianos incitan a los usuarios que los comparten y a sus seguidores a reconocer elementos de humanidad y potencial belleza moral. En otros términos, a comportarse de manera moralmente virtuosa. Pero, ¿de dónde surge la necesidad de exteriorizar sentimientos y proyectos felices valiéndose de los 
niños? Algunos estudios ya aluden a este sharenting lucrativo en el que, parece ser, la felicidad es un recurso mercadotécnico esencial (Fox \& Hoy, 2019; Siibak \& Traks, 2019; Peale, 2017).

A pesar del conocimiento originado en el ámbito científico con base en la idealización y proyección feliz de la vida cotidiana en redes sociales (Dale et al., 2019; Gorton \& Garde-Hansen, 2013; Freitas, 2017), nos hallamos en un entorno de cuestionable autoría parental (Choi \& Lewallen, 2017), donde no solo entran en escena la exposición excesiva de información personal de los niños (sharenting) (BlumRoss \& Livingstone, 2017; Kopecky, Szotkowski, Aznar-Díaz \& Romero-Rodríguez, 2020; Yebra, 2018), sino la insistencia de estas figuras paternales y maternales por expresar cuán divertidas, increíbles y geniales son las rutinas de estos. Se habla de instagramers que, en algunos casos, superan el millón de seguidores y generan interacciones propias a las de cualquier otro influencer.

Ante esta perspectiva de latente influencia e impacto mediático y, conscientes de la naturaleza multimodal de Instagram, se presenta, a continuación, una nueva línea de exploración que, desde el halo visual, compare la proporcionalidad de polaridad entre texto e imagen. De hecho, el escenario objeto de estudio en esta investigación se acogía a la narrativa subyacente en la red visual por antonomasia, extendiendo un nuevo itinerario de análisis en la comprensión de este fenómeno.

\section{Limitaciones y futuras líneas de investigación}

Dada la vertiente exploratoria del estudio, admitimos la limitación que supone la muestra y la estructuración esencialmente semántica acometida en el apartado metodológico. Si bien, apreciamos que el análisis de sentimiento proporcionado por estructuras computacionales machile learning puede considerarse un punto de partida para la investigación de los procesos del lenguaje natural en redes sociales, convirtiéndose en un instrumento esencial, innovador y compatible con otros métodos.

Por otro lado, y, considerando la internacionalización y posibilidades de análisis léxico en varios lenguajes proporcionados por estos sistemas, sugerimos la profundización en las motivaciones parentales que generan este fenómeno de idealización infantil en Instagram. Por una parte, planteamos la necesidad de confrontar los resultados con el análisis semiótico y visual de las fotografias en concurrencia con las entradas léxicas. Se trata, en este orden de cosas, de verificar la sinceridad de supuesta felicidad en estas cuentas desde la concepción multimodal, o si, por el contrario, son un reflejo construido e irreal de la cotidianeidad con carices publicitarios, auto-promocionales y/o lucrativos. Por otra, se requiere un estudio pormenorizado de la opinión de esta comunidad infantil y parental, que exponga y responda a los factores que hacen de este álbum en línea un entorno feliz. 


\section{Apoyos}

Este trabajo se ha elaborado en el marco de Alfamed (Red Interuniversitaria Euroamericana de Investigación en Competencias Mediáticas para la Ciudadanía), con el apoyo del Proyecto I+D+I (2019-2021), titulado «Youtubers e Instagrammers: La competencia mediática en los prosumidores emergentes» con clave RTI2018093303-B-I00, financiado por Ministerio de Ciencia, Innovación y Universidades de España y el Fondo Europeo de Desarrollo Regional (FEDER).

\section{Referencias bibliográficas}

American Psychological Association (2007). Task force on the sexualization of girls. Recuperado de https://bit.ly/2RFp5B6

American Psychological Association (2017). Ethical principles of psychologists and code of conduct. Recuperado de https://bit.ly/2iDuXKq

Ao, S. (2018). Sentiment analysis based on financial tweets and market information. En 2018 International Conference on Audio, Language and Image Processing (pp. 321-326). Shanghai: ICALIP. https://doi.org/10.1109/ICALIP.2018.8455771

Araque, O., Corcuera-Platas, I., Sánchez-Rada, F., y Iglesias, C.A. (2017). Enhancing deep learning sentiment analysis with ensemble techniques in social applications. Expert Systems with Applications, 77, 236-246. https://doi.org/10.1016/j.eswa.2017.02.002

Asch, S.E. (1946). Forming impressions of personality. The Journal of Abnormal and Social Psychology, 41(3), 258-290. https://doi.org/10.1037/h0055756

Ashok, M., Rajanna, S., y Joshi, P.V. (2016). A personalized recommender system using machine learning based sentiment analysis over social data. En 2016 IEEE Students' Conference on Electrical, Electronics and Computer Science (pp. 1-6). Bhopal, India: SCEECS. https://doi.org/10.1109/SCEECS.2016.7509354

Berezan, O., Krishen, A.S., Agarwal, S., y Kachroo, P. (2017). The pursuit of virtual happiness: Exploring the social media experience across generations. Journal of Business Research, 89, 455-461. https://doi.org/10.1016/j.jbusres.2017.11.038

Berlanga-Martínez, I., y Martínez-Rodrigo, E. (2010). Enl@ce, 7(2), 47-61. https://bit. ly/2UfNEr0

Blum-Ross, A., y Livingstone, S. (2017). Sharenting, parent blogging, and the boundaries of the digital self. Popular Communication, 15(2), 110-125. https://doi.org/10.1080/15405 702.2016.1223300

Bosangit, C., y Demangeot, C. (2016). Exploring reflective learning during the extended consumption of life experiences. Journal of Business Reserach, 69(1), 208-215. https:// doi.org/10.1016/j.jbusres.2015.07.033

Bourdieu, P. (1990). Photography: A middlebrow art. Standford: Stanford University Press.

Buijzen, M., Schuurman, J., y Bomhof, E. (2008). Associations between children's television advertising exposure and their food consumption patterns: A household diary-survey study. Appetite, 50, 231-239. https://doi.org/10.1016/j.appet.2007.07.006 
Calvo-González, S., y San-Fabián-Maroto, J.L. (2018). Selfies, jóvenes y sexualidad en Instagram: Representaciones del yo en formato imagen. Pixel-Bit, 52, 167-181. https:// doi.org/10.12795/pixelbit.2018.i52.12

Casado-Riera, C., y Carbonell, X. (2018). La influencia de la personalidad en el uso de Instagram. Aloma, 36(2), 23-31. Recuperado de https://bit.ly/37dFP9b

Chacón-López, H., Romero-Barriga, J.F., Aragón-Carretero, Y., y Caurcel-Cara, M.J. (2016). Construcción y validación de la escala de conductas sobre sexting (ECS). Revista Española de Orientación y Psicopedagogía, 27(2), 99-115. https://doi.org/10.5944/ reop.vol.27.num.2.2016.17116

Chae, J. (2018). Reexamining the relationship between social media and happiness: The effects of various social media platforms on reconceptualized happiness. Telematics and Informatics, 35(6), 1656-1664. https://doi.org/10.1016/j.tele.2018.04.011

Choi, G.Y., y Lewallen, J. (2017). Say Instagram, kids!: Examining sharenting and children's digital representations on Instagram. Howard Journal of Communication, 29(2), 144-164. https://doi.org/10.1080/10646175.2017.1327380

Dale, K.R., Raney, A.A., Ji, Q., Janicke-Bowles, S.H., Baldwin, J., Rowlett, J.T., ... Oliver, M.B. (2019). Self-transcendent emotions and social media: Exploring the content and consumers of inspirational Facebook posts. New Media \& Society, 1-21. https://doi. org/10.1177\%2F1461444819865720

De-Casas-Moreno, P., Tejedor-Calvo, S., y Romero-Rodríguez, L.M. (2018). Micronarrativas en Instagram: Análisis del storytelling autobiográfico y de la proyección de identidades de los universitarios del ámbito de la comunicación. Prisma Social, 20, 41-57. Recuperado de https://bit.ly/30EDEsT

Díaz-Bustamante, M., y Llovet-Rodriguez, C. (2017). Empowerment or impoverishment of children from social networks? Perceptions of sexualized images of girls in Instagram. El Profesional de la Información, 26(1), 77-87. https://doi.org/10.3145/epi.2017.ene.08

Diener, E. (2009). Happiness. En M.R., Leary y R.H. Hoyle (Eds.), Handbook of individual differences in social behavior (pp. 147-160). New York: Guilford. Recuperado de https://bit.ly/2sK57gh

Diessner, R., Solom, R.C., Frost, N.K., Parsons, L., y Davidson, J. (2008). Engagement with beauty: Appreciating natural, artistic, and moral beauty. The Journal of Psychology, 142(3), 303-332. https://doi.org/10.3200/JRLP.142.3.303-332

Diyanah-Abdullah, N.S., y Asnira-Zolkepli, I. (2017). Sentiment analysis of online crowd input towards brand provocation in Facebook, Twitter, and Instagram. En Proceedings of the International Conference on Big Data and Internet of Thing (pp. 67-74). New York: BDIOT. https://doi.org/10.1145/3175684.3175689

Domingo, M., Jewitt, C., y Kress, G. (2015). Multimodal social semiotics: Writing in online contexts. En J. Rowsell \& K. Pahl (eds.), The Routledge Handbook of Literacy Studies (pp. 251-266). London: Routledge. Recuperado de https://bit.ly/2sMlbOE

Dresner, E., y Herring, S.C. (2010). Functions of the nonverbal in CMC: Emoticons and 833 illocutionary force. Communication Theory, 20(3), 249-268. https://doi. org/10.1111/j.1468-2885.2010.01362.x 
Fox, A.K., y Hoy, M.G. (2019). Smart devices, smart decisions? Implications of parents' sharenting for children's online privacy: An investigation of mothers. Journal of Public Policy and Marketing, 38(4), 414-432. https://doi.org/10.1177/0743915619858290

Freitas, D. (2017). The happiness effect: How social media is driving a generation to appear perfect at any cost. Oxford: Oxford University Press.

Gómez-Espino, J.M., y Blanco-López, J. (2005). Los niños en la publicidad. Una propuesta de categorización de las representaciones sociales sobre la infancia en los anuncios televisivos. ZER Revista de Estudios de Comunicación, 10(19), 53-79. Recuperado de https://bit.ly/36iU2jM

Gorton, K., y Garde-Hansen, J. (2013). Social media, happiness, and virtual communities. En Emotion online (pp. 103-125). London: Palgrave Macmillan. https://doi. org/10.1057/9781137312877_5

Hadjipanayis, A., Efstathiou, E., Altorjai, P., Stiris, T., Valiulis, A., Koletzko, B., y Fonseca, H. (2019). Social media and children: What is the paediatrician's role? European Journal of Pediatrics, 178(10), 1605-1612. https://doi.org/10.1007/s00431-019-03458-w

Hasan, A., Moin, S., Karim, A., y Shamshirband, S. (2018). Machine learning-based sentiment analysis for Twitter accounts. Mathematical and Computational Applications, 23(11), 1-15. https://doi.org/10.3390/mca23010011

Ho, H., Shin, W., y Lwin, M.O. (2017). Social networking site use and materialistic values among youth: The safeguarding role of the parent-child relationship and self-regulation. Communication Research, 46(8), 1119-1144. https://doi. org/10.1177/0093650216683775

Huffaker, D. (2010). Dimensions of leadership and social influence in online 853 communities. Human Communication Research, 36(4), 593-617. https://doi.org/10.1111/j.14682958.2010.01390.x

Hummel-O'Donnell, N. (2018). Storied lives on Instagram: Factors associated with the need for personal-visual identity. Visual Communication Quarterly, 25(3), 131-142. https:// doi.org/10.1080/15551393.2018.1490186

Instagram (2020). About us. Recuperado de https://bit.ly/38n6mBa

Izquierdo-Iranzo, P., y Gallardo-Echenique, E. (2020). Estudigramers: Influencers del aprendizaje. [Studygrammers: Learning influencers]. Comunicar, 62, 115-125. https://doi. org/10.3916/C62-2020-10

Kim, Y., y Kim, J.H. (2018). Using computer vision techniques on Instagram to link users' personalities and genders to the features of their photos: An exploratory study. Information Processing and Management, 54, 1101-1114. https://doi.org/10.1016/j. ipm.2018.07.005

Kopecky, K., Szotkowski, R., Aznar-Díaz, I., y Romero-Rodríguez, J. M. (2020). The phenomenon of sharenting and its risks in the online environment. Experiences from Czech Republic and Spain. Children and Youth Services Review, 110. https://doi. org/10.1016/j.childyouth.2020.104812

Krippendorff, K. (1980). Metodología de análisis de contenido. Teoría y práctica. Barcelona: Paidós. 
Larrañaga, E., Navarro, R., y Yubero, S. (2018). Socio-cognitive and emotional factors on perpetration of cyberbullying. [Factores socio-cognitivos y emocionales en la agresión del ciberacoso]. Comunicar, 56, 19-28. https://doi.org/10.3916/C56-2018-02

Lee, E., Lee, J.A., Moon, J.H., y Sung, Y. (2015). Pictures speak louder than words: Motivations for using Instagram. Cyberpsychology, Behavior and Social Networking, 18(9), 552556. https://doi.org/10.1089/cyber.2015.015

Le-Moignan, E., Lawson, S., Rowland, D.A., Mahoney, J., y Briggs, P. (2017). Has Instagram fundamentally altered the 'family snapshot'? Technology in Households, 6(11), 4935-4947. https://doi.org/10.1145/3025453.3025928

Liu, B. (2015). Sentiment analysis: Mining opinions, sentiments, and emotions. Cambridge: Cambridge University Press.

Livingstone, S., Mascheroni, G., Ólafsson, K., y Haddon, L. (2014). Children's online risks and opportunities: Comparative findings from EU Kids online and Net Children Go Mobile. London: EU Kids Online. Recuperado de https://bit.ly/37ffDXo

Lup, K., Trub, L., y Rosenthal, L. (2015). Instagram \#instasad?: Exploring associations among Instagram use, depressive symptoms, negative social comparison, and strangers followed. Cyberpsychology, Behavior and Social Networking, 18(5), 247-252. https://doi. org/10.1089/cyber.2014.0560

Matijaščć, A.S. (2018). The right to privacy and publishing of personal data, information (about) and photographs of minor children. Glasilo Future, 1(3), 27-59.

Marinas, L. (2019). Instagram: Donde Millenialls, Generación Z, McLuhan y Bolter se cruzan. Cuadernos de Información y Comunicación, 24, 187-201. https://doi. org/10.5209/ciyc.64641

Micu, A., Micu, A.E., Geru, M., y Lixandroiu, R.C. (2017). Analyzing user sentiment in social media: Implications for online marketing strategy. Psychology \& Marketing, 34(12), 1094-1100. https://doi.org/10.1002/mar.21049

Nathanson, A.I. (2004). Factual and evaluative approaches to modifying children's responses to violent television. Journal of Communication, 54, 321-336. https://doi.org/10.1111/ j.1460-2466.2004.tb02631.x

Pang, B., Lee, L., y Viathyanathan, S. (2002). Thumbs up?: Sentiment classification using machine learning techniques. En Proceedings of the ACL-02 conference on empirical methods in natural language processing (pp. 79-86). Stroudsburg: Association for Computational Linguistics.

Peale, N.V. (2017). El poder del pensamiento positivo. México: Oceano.

Peña-Cárdenas, F., Rojas-Solís, J.L., y García-Sánchez, P.V. (2018). Uso problemático de Internet, cyberbullying y ciber-violencia de pareja en jóvenes universitarios. Diversitas: Perspectivas en Psicología, 14(2), 205-219. https://doi.org/10.15332/s1794-9998

Reinecke, L., y Trepte, S. (2014). Authenticity and well-being on social network sites: A two wave longitudinal study on the effects of online authenticity and the positivity bias in SNS communication. Computers in Human Behavior, 30(1), 95-102. https://doi. org/10.1016/j.chb.2013.07.030

Rose, G. (2010). Doing family photography: The domestic, the public and the politics of sentiment. Surrey: Ashgate Publishing. https://doi.org/10.4324/9781315577890 
Scheinbaum, A. (2017). The dark side of social media: A consumer psychology perspective. New York: Routledge. https://doi.org/10.4324/9781315167718

Siibak, A., y Traks, K. (2019). The dark sides of sharenting. Catalan Journal of Communication and Cultural Studies, 11(1), 115-121. https://doi.org/10.1386/cjcs.11.1.115_1

Thurlow, C., Lengel, L., y Tomic, A. (2004). Computer mediated communication: Social interaction and the Internet. London: Sage. https://doi.org/10.5860/choice.42-0992

UNESCO (2020). La atención y educación de la primera infancia. UNESCO. https://bit. ly/39eFTFT

Veenhoven, R. (1991). Is happiness relative? Social Indicators Research, 24(1), 1-34. https:// doi.org/10.1007/BF00292648

Vizcaíno-Verdú, A., Contreras-Pulido, P., y Guzmán-Franco, M.D. (2019). Lectura $\mathrm{y}$ aprendizaje informal en YouTube: El booktuber. [Reading and informal learning trends on YouTube: The booktuber]. Comunicar, 59, 95-104. https://doi. org/10.3916/C59-2019-09

Waterloo, S.F., Baumgartner, S.E., Peter, J., y Valkenburg, P.M. (2018). Norms of online expressions of emtoion: Comparing Facebook, Twitter, Instagram, and Whatsapp. New Media E Society, 20(5), 1813-1831. https://doi.org/10.1177\%2F1461444817707349

Wernholm, M., y Reneland-Forsman, L. (2019). Children's representation of self in social media communities. Learning, Culture and Social Interaction, 23, 1-15. https://doi. org/10.1016/j.lcsi.2019.100346

Willoughby, M. (2018). A review of the risks associated with children and young people's social media use and the implications for social work practice. Journal of Social Work Practice, 33(2), 127-140. https://doi.org/10.1080/02650533.2018.1460587

Yebra, J.A. (2018). El régimen de prestación del consentimiento para la intromisión en los derechos de la personalidad de los menores. Especial referencia al fenómeno del sharenting. Actualidad Jurídica Iberoamericana, 8, 253-264. https://bit.ly/2UgUCM9

Zhan, M., Tu, R., y Yu, Q. (2018). Understanding readers: Conducting sentiment analysis of Instagram captions. En Proceedings Of 2018 The 2nd International Conference on Computer Science and Artificial Intelligence (pp. 33-40). Shenzhen, China: Association for Computing Machinery. https://doi.org/10.1145/3297156.3297270 